\title{
Graphitic Interfacial Layer to Carbon Nanotube for Low Electrical Contact Resistance
}

\author{
Yang Chai ${ }^{\dagger \neq}$ Arash Hazeghi ${ }^{\dagger}$, Kuniharu Takei ${ }^{*}$, Hong-Yu Chen ${ }^{\dagger}$, Philip C. H. Chan ${ }^{\ddagger \S}$, Ali Javey ${ }^{*}$ and \\ H. -S. Philip Wong ${ }^{\dagger}$
}

${ }^{\dagger}$ Department of Electrical Engineering and Center for Integrated Systems, Stanford University, USA

${ }^{\star}$ Department of Electronic and Computer Engineering, Hong Kong University of Science and Technology, Hong Kong, China

${ }^{*}$ Department of Electrical Engineering and Computer Sciences, University of California at Berkeley, USA

${ }^{\S}$ Department of Electronic and Information Engineering, The Hong Kong Polytechnic University, Hong Kong, China

Tel.: (1)-650-283-0290, Email: yangchai@stanfrord.edu

\begin{abstract}
Graphitic interfacial layer is used to wet the surface of carbon nanotube and dramatically lower the contact resistance of metal to metallic single-wall carbon nanotube (m-CNT). Using Ni-catalyzed graphitization of amorphous carbon (a-C), the average resistance of metal $/ \mathrm{m}-\mathrm{CNT}$ is reduced by $7 \mathrm{X}$ compared to the same contact without the graphitic layer. Small-signal conductance measurements from $77 \mathrm{~K}$ to $300 \mathrm{~K}$ reveal the effective contact improvement.
\end{abstract}

\section{Introduction}

CNT is a promising both as interconnect $[1,2]$ and transistor [3]. Electrical contact is an indispensable part in integrated circuit. The small contact area makes the electrical coupling between CNT and metal electrode extremely difficult. Experimental results have shown that the contact resistance is still large even for the CNT with metallic band-structure, and the contact resistance is diameter-dependent $[4,5]$. This contact resistance possibly results from the non-wetting tubular structure and non-clean interface between CNT and metal (Fig. 1(a)). There is an atomic-level separation between CNT and metal for non-wetting surfaces. In this work, we used amorphous carbon (a-C) as an interfacial layer between $\mathrm{CNT}$ and metal electrode. The a-C was graphitized at high temperature catalyzed by carbon-soluble Ni $[6,7]$. We studied the electrical transport through the same CNT device with and without graphitic interfacial layer using small-signal conductance measurement at low temperature.

\section{Device Fabrication}

Fig. 2 shows the process flow for fabricating the test structure. Horizontally aligned single walled CNTs (200 $\mu \mathrm{m}$ long) were grown on quartz substrate using methane and Fe catalyst [8]. The diameter of the CNT is $1.2 \pm 0.3 \mathrm{~nm}$. The CNTs were then transferred to a $\mathrm{Si} / \mathrm{SiO}_{2}$ substrate [8]. Then a $2.5 \mathrm{~nm}$-thick a-C layer was deposited by e-beam evaporation on top of the CNT and patterned by lithography. $\mathrm{Au} / \mathrm{Ni}$ metal contact was subsequently patterned on the a-C surface by liftoff process. The channel length of the CNT device was defined by standard photolithography. The CNT device length studied here is $1 \mu \mathrm{m}$ between the two metal electrodes. A control structure without a-C interfacial layer was also fabricated on the same CNT. Unwanted CNT on the substrate were etched by oxygen plasma. The average density of CNT is $3-5$ $\mathrm{CNT} / \mu \mathrm{m}$, thus there are $\sim 1-3 \mathrm{CNTs}$ per $1 \mu \mathrm{m}$-width device. The fabricated samples were annealed at $750{ }^{\circ} \mathrm{C}$ for $10 \mathrm{~min}$ in hydrogen. After the anneal, the a-C interfacial layer is graphitized, assisted by the carbon-soluble Ni (Fig. 1(b)).
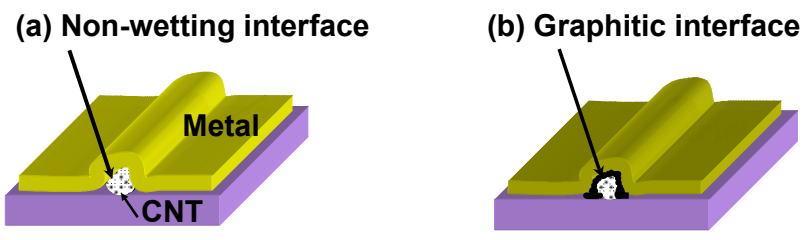

\section{Side-contact configuration}

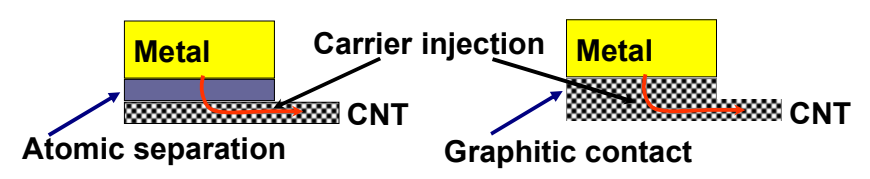

Fig. 1. Schematic of the interface between CNT and metal electrode. (a) A finite separation caused by non-wetting or non-clean metal/CNT interface. A barrier forms between the metallic CNT and metal electrode. (b) The graphitic interface has similar bonding to CNT, extending effective wave-function overlap, improving the wetting and enlarging the contact area.
(1)

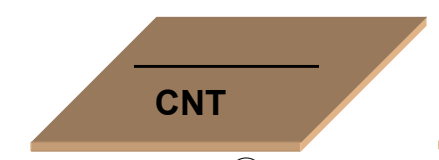

(3)

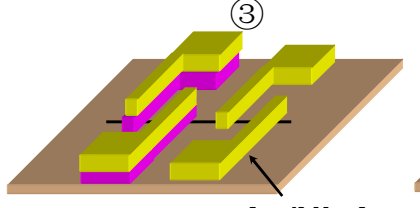

Au/Ni Annealing: Graphitic carbon
Fig. 2. Process flow for fabricating the test structure. The metal pads with and without graphitic interfacial layer were fabricated on the same CNT (200 $\mu \mathrm{m}$ long). The a-C layer was transformed to graphitic carbon by a high temperature annealing process catalyzed by $\mathrm{Ni}$. 


\section{Material Characterization}

The transformation from a-C to graphitic carbon, assisted by the e-beam irradiation inside an SEM or TEM [9, 10], has been used to improve the contact between multi-walled CNT and metal. However this transformation involves the high local energy and temperature. Ref. [6] shows that the presence of a carbon-soluble catalyst (e.g. Ni) effectively improves this transformation with an annealing process. This work capitalizes on this observation for a wafer-scale contact improvement technology. Fig. 3 shows the Raman spectra of the as-deposited a-C layer and the Ni-catalyzed carbon layer after the annealing process. The sharp $G\left(1571 \mathrm{~cm}^{-1}\right)$ peak indicates the successful conversion from the a-C to the graphitic carbon. This graphitic carbon has similar bonding as the carbon of the CNT, extending effective wave function overlap for conduction band electrons in the form of $\mathrm{P}_{\mathrm{z}}-\mathrm{P}_{\mathrm{z}}$ covalent bonding. The high temperature annealing process also improves the metal wetting to CNT, and increases the actual contact area.
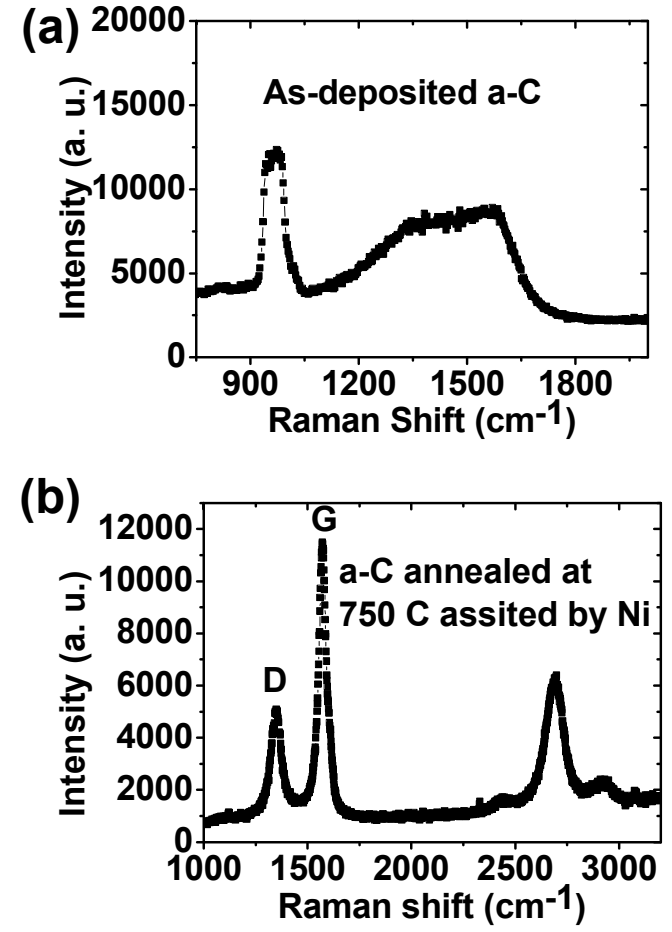

Fig. 3. Raman spectra of (a) as-deposited a-C layer, showing broad peak distribution. (b) a-C layer annealed at $750{ }^{\circ} \mathrm{C}$ with hydrogen flow for $10 \mathrm{~min}$. The sharp $\mathrm{G}\left(1571 \mathrm{~cm}^{-1}\right)$ peak indicates the successful transformation from the a-C to the graphitic carbon. The Ni catalyst effectively improves the graphitization.

\section{Electrical Results}

Fig. 4 shows SEM images of the fabricated device. To ensure a direct comparison, the same $200 \mu \mathrm{m}$ long CNT was used for both the devices with and without graphitic interfacial layer metal electrode. The CNTs extend beyond the electrodes and make side contacts to the electrodes.

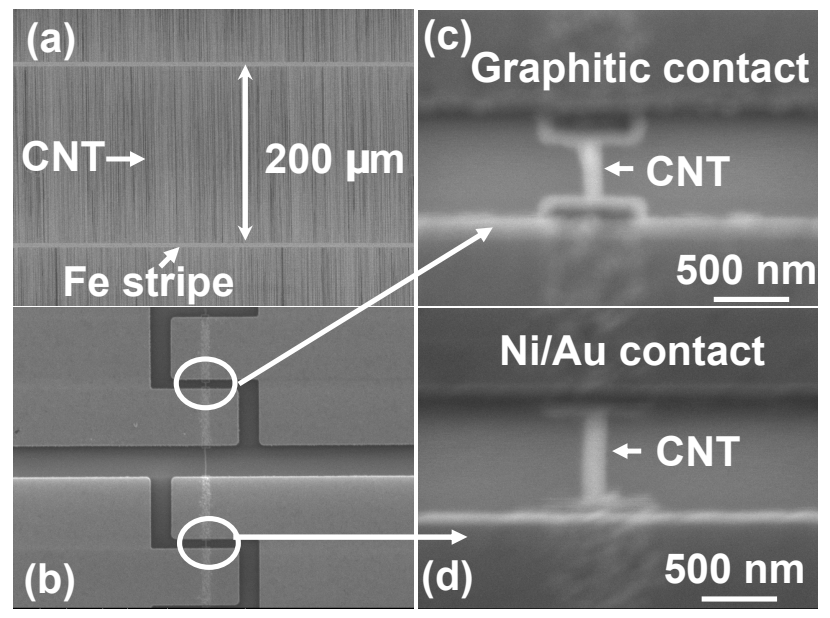

Fig. 4. SEM images of (a) $200 \mu \mathrm{m}$ long horizontally aligned CNT, (b) a test structure on the same CNT, (c) the CNT with graphitic interfacial layer, and (d) $\mathrm{Au} / \mathrm{Ni}$ pad only without the graphitic interfacial layer.

Fig. 5 shows the typical I-V curves of the same CNT with and without graphitic interfacial layer. The as-synthesized CNTs are single-wall CNTs and consist of a mixture of semiconducting CNT and metallic CNT. We selected the CNT devices without back-gate modulation (inset of Fig. 5) and measured the resistance for the different samples in a two-point configuration. The drive current in the CNT device increase dramatically after introducing the graphitic interfacial layer.

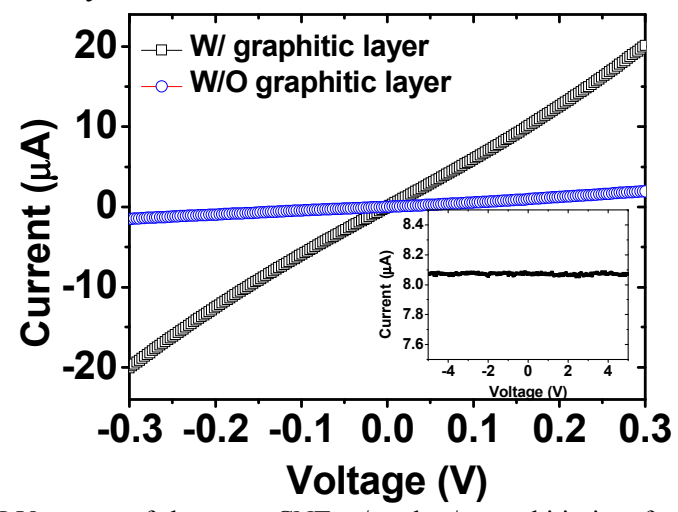

Fig. 5. I-V curves of the same CNT w/ and w/o graphitic interfacial layer. The CNT w/ graphitic layer shows more drive current than that w/o graphitic layer. Inset shows the current as a function of the back-gate voltage. The CNT has weak gate modulation over a large gate voltage scan, indicating the metallic-like CNT.

Fig. 6 shows the histogram of the conductance distribution for 59 metallic CNT devices. The conductance is greatly improved after the introduction of the graphitic layer. Since the devices with and without graphitic interfacial contacts are fabricated on the same CNT, the improvement is a direct result of efficient bonding between the CNT and the graphitic layer. It is well-known that traditional 4-terminal 
measurements for extracting contact resistance do not yield accurate results for 1-D systems such as a single m-CNT with small density of states and semi-ballistic transport. The metal contacts disturb the local channel potential at the point of contact with CNT and therefore an accurate voltage drop cannot be measured [11]. The CNT without graphitic interfacial layer shows larger resistance and the resistance is bias-dependent, indicating a contact barrier between the CNT and metal (Fig. 7).

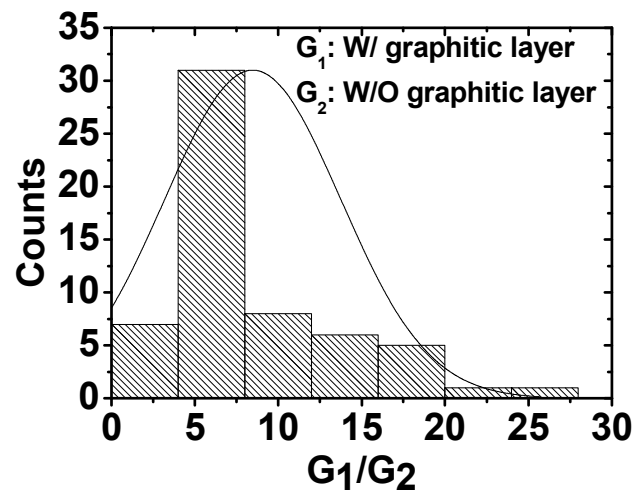

Fig. 6. Histogram of the conductance distribution from 59 working m-CNT devices. The conductance of the CNT was extracted by two-point measurement. The conductance of the CNT with graphitic layer was normalized over that of the CNT without graphitic layer. The conductance increase results from the electrical contact improvement by the introduction of the graphitic interfacial layer.

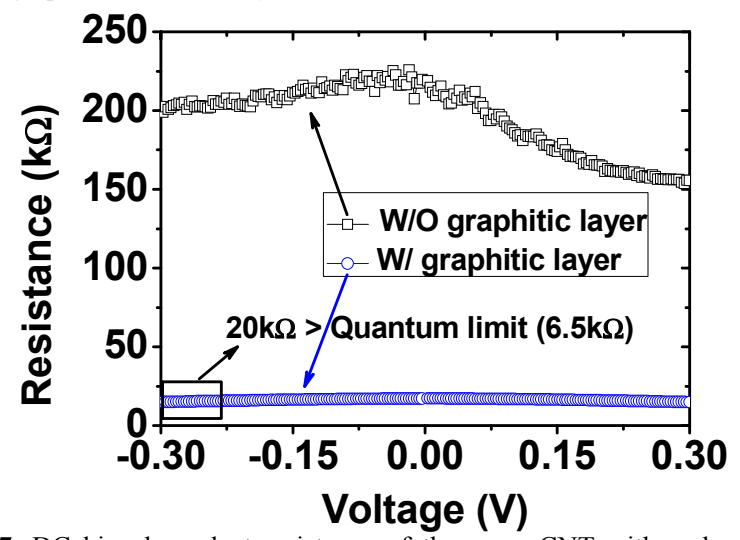

Fig. 7. DC bias-dependent resistance of the same CNT with and without graphitic interfacial layer. The large dependency of the resistance of the CNT w/o graphitic layer suggests the existence of the contact barrier. Measured lower resistance $(20 \mathrm{~K} \Omega)$ is close to but still higher than the quantum conductance limit $(6.5 \mathrm{~K} \Omega)$. This is due to scattering (diffusive transport) inside the channel and residual barrier at the contacts.

A lock-in amplifier setup (Fig. 8) was used to measure zero-bias small-signal conductance of the metal $/ \mathrm{m}-\mathrm{CNT}$ contact more accurately. Fig. 9 shows the conductance dispersion as a function of the signal amplitude. The conductance of CNT device without graphitic interfacial layers shows large dispersion as a function of the signal amplitude. This non-linear effect is indicative of a transport barrier between m-CNT and metal contact. In contrast, the conductance of the CNT device with graphitic contact shows little dependence on the signal amplitude, indicating Ohmic-like contact. This provides additional evidence of the $\mathrm{CNT} /$ metal contact improvement by graphitic interfacial layer. Fig. 10 shows Arrehenius plot of the same CNT with and without graphitic layer. The CNT device with the graphitic interfacial layer shows less temperature dependence.

\section{Controller PC with GPIB}

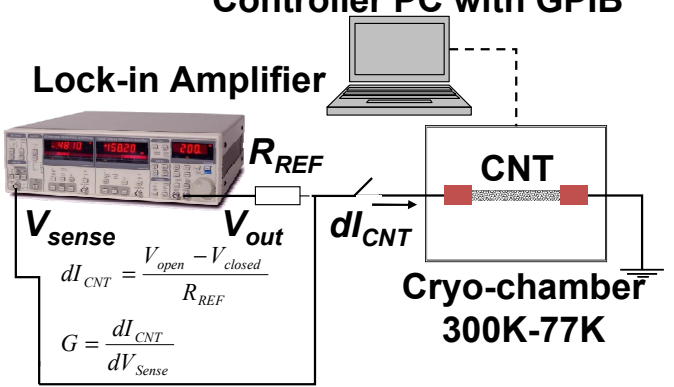

Fig. 8. Schematics of experimental setup for small-signal conductance measurement using a lock-in amplifier and a cryogenic probe station. Signal is sampled with switch S1 open and then closed. Conductance of the DUT can be calculated using this difference and $\mathrm{R}_{\mathrm{REF}}$.
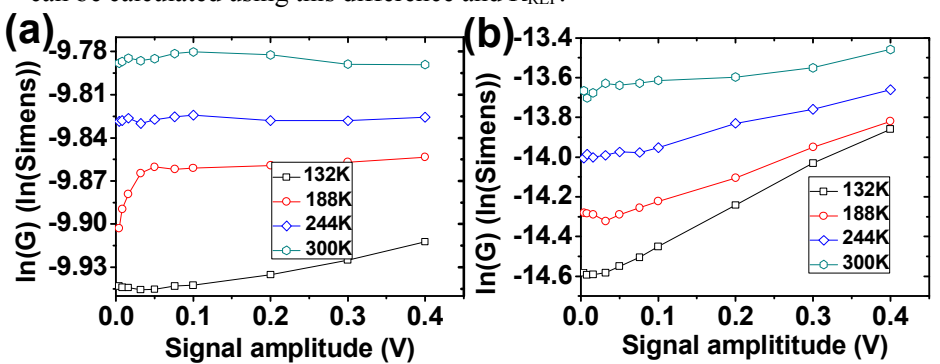

Fig. 9. Small signal conductance of the same CNT as a function of the signal amplitude. The same CNT (a) with and (b) without graphitic interfacial layer. The CNT without graphitic interfacial layer shows large signal amplitude dependency, indicating the large barrier between the CNT and metal electrode.

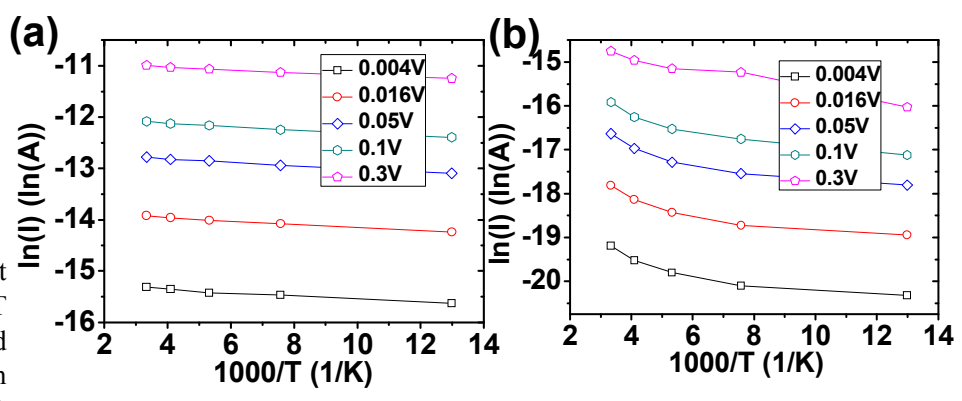

Fig. 10. Arrhenius plots of the same CNT (a) with and (b) without graphitic interfacial layer at 5 different signal amplitudes. The CNT without graphitic interfacial layer shows large temperature dependency, indicating the large barrier between the CNT and metal electrode.

Fig. 11 shows the extracted effective activation energies for the same CNT with $(36 \mathrm{meV})$ and without $(204 \mathrm{meV})$ graphitic layer. Fig. 12 shows conductance improvement of the same device over the one without graphitic interfacial layer as a function of the signal amplitude and the temperature. The conductance was greatly increased for all 6 measured devices by the introduction of the graphitic interfacial layer. 


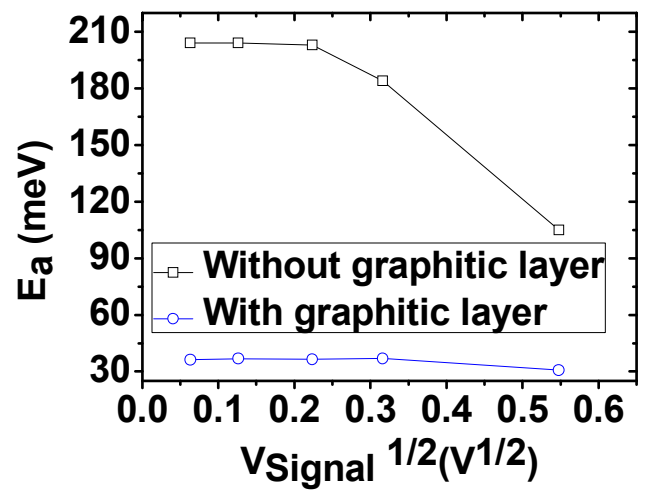

Fig. 11. Effective activation energies as a function of square root of signal amplitude for the same CNT device w/ and w/o graphitic interfacial layer. The effective activation energies ware extracted according to the Arrhenius plots. The activation energies for the CNT device w/ and w/o graphitic interfacial layer are $36 \mathrm{meV}$ and $204 \mathrm{meV}$ at zero bias, respectively.

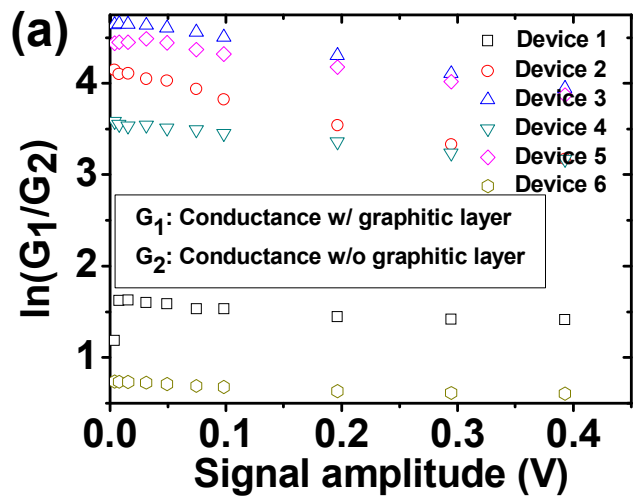

(b)

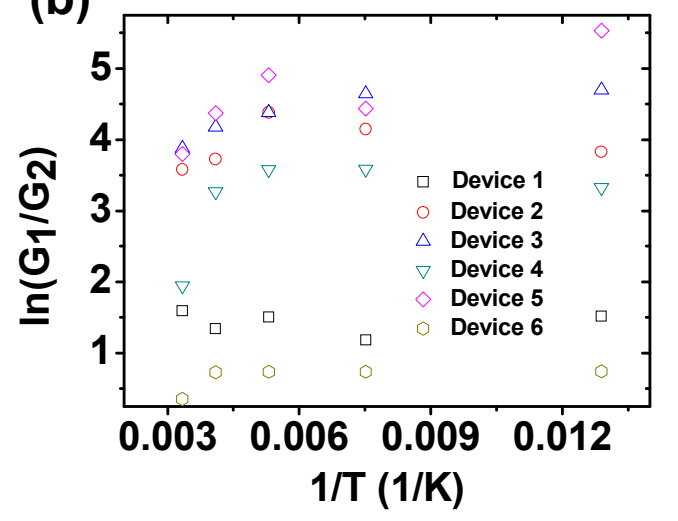

Fig. 12. Conductance improvement of the same device with graphitic interfacial layer over the one without the layer as a function of (a) signal amplitude and (b) temperature. Although the conductance varies for different devices due to the variations from fabrication process and CNT diameter, the conductance is noticeably improved for all measured devices.

\section{Conclusion}

Metal to metallic CNT contact resistance is dramatically improved by a graphitic interfacial layer. The graphitic layer is formed by Ni-catalyzed dissolution of a-C in the metal. This opens up a new pathway toward Ohmic contact between metal and metallic CNT and may point to a way to form Ohmic contact for semiconducting CNT as well.

\section{Acknowledgement}

The authors thank Mr. Jason Matthew Parker for his help with the annealing process. This work is supported in part by FENA, one of six research centers funded under the Focus Center Research Program (FCRP), a Semiconductor Research Corporation subsidiary, the Research Grant Council of Hong Kong Government under CERG grant HKUST 611307, and the Berkeley Sensors and Actuators Center (BSAC).

\section{References}

[1] G. Close, S. Yasuda, B. C. Paul, S. Fujita and H. -S. P. Wong, "Measurement of subnanosecond delay through multiwall carbon nanotubelocal interconnect in a CMOS integrated circuit." IEEE T-ED, Vol. 56, No. 1, pp. 43-49, January 2009.

[2] Y. Chai and P. C. H. Chan, "High electromigration-resistance of copper/carbon nanotube composite for interconnect application," IEEE IEDM Technical Digest, pp. 607-610, December 2008.

[3] L. Wei, D. J. Frank, L. Chang and H. -S. P. Wong, "A non-iterative compact model for carbon nanotube FETs incorporating source exhaustion effect," IEEE IEDM Technical Digest, pp. 917-920, December 2009.

[4] W. Kim, A. Javey, R. Tu, J. Cao, Q. Wang, and H. J. Dai, "Electrical contacts to carbon nanotubes down to $1 \mathrm{~nm}$ in diameter," Appl. Phys. Lett., Vol. 87, pp. 173101-173103, October 2005.

[5] Y. Chai, Z. Y. Xiao, and P. C. H. Chan, "Horizontally aligned carbon nanotube bundles for interconnect application: diameter-dependent contact resistance and mean free path," Nanotechnology, Vol. 21, pp.235705-235709, May 2010.

[6] M. Zheng, K. Take, B. Hsia, H. Fang, X. B. Zhang, N. Ferralis, H. Ko, Y. L. Chueh, Y. G. Zhang, R. Maboudian and A. Javey, "Metal-catalyzed crystallization of amorphous carbon to graphene," Appl. Phys. Lett., Vol. 96, pp. 063110-063112, February 2010.

[7] T. Itoh, "Study of reactions in transition metal/amorphous carbon layered thin films using transmission electron microscopy," Stanford PhD thesis, 1996.

[8] N. Patil, A. Lin, E. R. Myers, K. Ryu, A. Badmave, C. W. Zhou, H-. S. P. Wong and S. Mitra, "Wafer-scale growth and transfer of aligned single-walled carbon nanotubes," IEEE Trans. Nanotechnology, Vol. 8, No. 4, pp. 498-504, July 2009.

[9] Q. Chen, S. Wang and L. M. Peng, "Establishing Ohmic contacts for in situ current-voltage characteristic measurements on a carbon nanotube inside the scanning electron microscope," Nanotechnology, Vol. 17, pp. 1087-1098, January 2006.

[10] K. Rykaczewski, M. R. Henry and A. G. Fedorov, "Electron beam induced deposition of residual hydrocarbons in the presence of a multiwall carbon nanotube," Appl. Phys. Lett., Vol. 95, No. 11, pp. 113112-113114, September 2009.

[11] S. Datta, Quantum Transport Atom to Transistors, Cambridge University Press, 2005. 